\title{
Renormalization of the one-pion-exchange interaction
}

\author{
T. Frederico, ${ }^{\mathrm{a}}$ V.S. Timóteo, ${ }^{\mathrm{b}}$ Lauro Tomio $^{\mathrm{c}}$ \\ ${ }^{a}$ Departamento de Física, Instituto Tecnológico de Aeronáutica, CTA, \\ 12228-900, São José dos Campos, Brasil \\ ${ }^{\mathrm{b}}$ Nuclear Theory and Elementary Particle Phenomenology Group, \\ Instituto de Física, Universidade de São Paulo, 05315-970, São Paulo, Brasil \\ ${ }^{\mathrm{c} I n s t i t u t o ~ d e ~ F i ́ s i c a ~ T e o ́ r i c a, ~ U n i v e r s i d a d e ~ E s t a d u a l ~ P a u l i s t a, ~ 01405-900, ~ S a ̃ o ~}$ \\ Paulo, Brasil
}

\begin{abstract}
A renormalization scheme for the nucleon-nucleon (NN) interaction based on a subtracted T-matrix equation is proposed and applied to the one-pion-exchange potential supplemented by contact interactions. The singlet and triplet scattering lengths are given to fix the renormalized strengths of the contact interactions. With only one scaling parameter $(\mu)$, the results show an overall very good agreement with neutron-proton data, particularly for the observables related to the triplet channel. The agreement is qualitative in the ${ }^{1} S_{0}$ channel. Between the low-energy NN observables we have examined, the mixing parameter of the ${ }^{3} S_{1}-{ }^{3} D_{1}$ states is the most sensible to the scale. The scheme is renormalization group invariant for $\mu \rightarrow \infty$.
\end{abstract}

PACS numbers: 21.30.Fe, 13.75.Cs, 11.10.Gh, 03.65.Nk, 21.45.+v.

Key words: N-N effective interactions, renormalization, nonrelativistic scattering theory, few-body

\section{Introduction}

The effective field theory (EFT) of nuclear forces based on a chiral expansion of an effective Lagrangian, as proposed by Weinberg [1], gives a nucleon-nucleon (NN) interaction in leading order which is the one-pion-exchange potential (OPEP) plus a Dirac-delta. He suggested to infer the values of the strength of the delta force in the ${ }^{1} S_{0}$ and ${ }^{3} S_{1}$ channels from the singlet and triplet scattering lengths respectively. Also such potential should be valid for momenta well below a typical momentum scale of quantum chromodynamics such 
as the rho meson mass $\left(m_{\rho} \sim 4 \mathrm{fm}^{-1}\right)$ [1]. Therefore, the intermediate virtual propagation of the NN system should be cut at the momentum scale of this order.

In spite of the significative results obtained by several authors, when implementing the EFT program for the two nucleon system [2-8], and also by a recent related approach [9], as far as we know the predictibility power of the leading order term (OPEP plus delta) with a single renormalization momentum scale was not fully explored in the literature. The result of such calculation would give a sound basis for the renormalization program of EFT in NN system. The leading order term was already considered in NN calculations, when comparing with more realistic calculations [10], or as part of interactions used in the EFT program $[3,4,11,12]$. However, we argue that, without a systematic analysis of the physical contribution coming from each order term in the renormalization procedure, it is easy to miss their real significance when parametrizing the full interaction to obtain the desired observables. To start, it is relevant to know how much physical information can one extract from the leading order term, using a renormalization procedure. What are the observables one can better describe with just the leading order term (the higher order terms should not affect too much these observables) and what are the observables for which higher order terms are essential? Our aim in this paper is to answer such questions. We hope the following analysis can be useful in the renormalization program of the effective field theory applied to the nucleon-nucleon system.

In this work we have verified strictly the validity of Weinberg suggestion, to first consider (in leading order) an effective potential which "consists of just a conventional one-pion exchange term, plus a direct neutron-proton $(n-p)$ interaction produced by the fourfermion terms" [1]. Such "four-fermion terms" consist of two delta-function interactions: one in the ${ }^{1} S_{0}$ channel and the other in the ${ }^{3} S_{1}$ channel. To be consistent with the original suggestion, to cut the virtual propagation of the NN system at the scale $m_{\rho}$, we first use a renormalization scale of such order. However, we understand this as a qualitative suggestion, such that we explore its limitation in the calculation of several low-energy NN observables. So, we finally consider a free renormalization scale in our numerical calculation, and try to obtain a consistent description, in order that such scale vanishes as a physical parameter as required by a renormalization group invariant theory.

In our approach, we use a renormalization scheme for the T-matrix, which is appropriate when considering the OPEP plus a delta potential. It incorporates the momentum scale $(\mu)$ where the two-nucleon propagation is removed and the scattering lenghts fix the renormalized strengths. A subtracted T-matrix integral equation is derived, where the subtraction is done at energy of $-\mu^{2}$, in the center of mass system (in units of $\hbar=1$ and nucleon mass $m=1$ ). The input is the T-matrix at energy $-\mu^{2}$, which is chosen as the OPEP supplemented by delta interactions. The scale $\mu$ is of the order of $m_{\rho}$, and we will show that the results for the renormalized T-matrix, in the limit of $\mu \rightarrow \infty$, once the scattering lengths are kept fixed, are quite consistent with $\mu \sim m_{\rho}$, at least for the diagonal components of the matrix (the extension of this statement will be clarified). As we verified, the numerical results in the ${ }^{3} S_{1}-{ }^{3} D_{1}$ channels, obtained by following this procedure are in good qualitative agreement with the experimental data for the $n-p$ 
phase-shifts, mixing parameter, the deuteron binding energy and D/S ratio, at laboratory energies below $\sim 50 \mathrm{MeV}$.

The higher order terms in the EFT expansion of the effective interaction will become more important for laboratory energies above $50 \mathrm{MeV}$ in the ${ }^{3} S_{1}-{ }^{3} D_{1}$ channels, where the leading order results clearly deviate from the data. However, in the ${ }^{1} S_{0}$ state the importance of the higher order terms appears already in the value of the effective range, which is underestimated in lowest order. Such results confirm that the effective interaction in the singlet state needs higher order correction (as already expected), but such corrections should not affect in an essential way the triplet states (particularly at low energies), which are already in a fair agreement with data.

In our scheme, it is considered an unregulated effective power expansion of the NN potential only in the mid- and short- range parts of the interaction, such that the well stablished long-range part (OPEP) is kept intact $\square$. So, the matrix elements of the full effective NN interaction, in the relative momentum space, will be given by

$$
\begin{aligned}
\left\langle\overrightarrow{p^{\prime}}\left|V_{E F T}\right| \vec{p}\right\rangle=\left\langle\overrightarrow{p^{\prime}}\left|V_{\pi}\right| \vec{p}\right\rangle & +\frac{1}{2 \pi^{2}}\left[\frac{1-\vec{\tau}_{1} \cdot \vec{\tau}_{2}}{4}\left(\lambda_{t}^{(0)}+\lambda_{t}^{(1)} p^{2}+\lambda_{t}^{(1) *} p^{2}+\cdots\right)+\right. \\
& \left.+\frac{3+\vec{\tau}_{1} \cdot \vec{\tau}_{2}}{4}\left(\lambda_{s}^{(0)}+\lambda_{s}^{(1)} p^{2}+\lambda_{s}^{(1) *} p^{\prime 2}+\cdots\right)\right]
\end{aligned}
$$

where the unregulated strengths of the contact interaction, and the higher order derivatives, have subindices $t$ and $s$, for the isospin singlet (spin triplet) and isospin triplet (spin singlet) channels respectively. The matrix element of the one-pion-exchange potential is given by:

$$
\left\langle\overrightarrow{p^{\prime}}\left|V_{\pi}\right| \vec{p}\right\rangle=-\frac{g_{a}^{2}}{4(2 \pi)^{3} f_{\pi}^{2}} \vec{\tau}_{1} \cdot \vec{\tau}_{2} \frac{\overrightarrow{\sigma_{1}} \cdot\left(\overrightarrow{p^{\prime}}-\vec{p}\right) \vec{\sigma}_{2} \cdot\left(\overrightarrow{p^{\prime}}-\vec{p}\right)}{\left(\overrightarrow{p^{\prime}}-\vec{p}\right)^{2}+m_{\pi}^{2}}
$$

where $\sigma_{i}$ and $\tau_{i}$ are the usual spin and isosping Pauli matrices for nucleon $i ; g_{a}(=1.25)$ is the axial coupling constant, $f_{\pi}(=93 \mathrm{MeV})$ is the pion weak-decay constant, and $m_{\pi}(=138 \mathrm{MeV})$ is the pion mass. $\vec{p}^{\prime}-\vec{p}$ is the momentum transfer. The leading order term of the expansion, which we are going to consider in the next sections, imply to take $\lambda_{s(t)}^{(n)}=0$ for all $n \geq 1$ in eq. (1).

In section II we present the formalism for the subtracted T-matrix, which is applied to the leading order term of the NN interaction. In section III we present our main results and conclusions.

1 The OPEP is common to all realistic interactions, and an effective power expansion which does not consider it will have to face difficulties with the parametrization, as the constants should represent simultaneously long and short range physics. 


\section{Subtracted T-matrix and the leading order NN interaction}

In the next we introduce our formalism which is based on a subtracted equation for the T-matrix. We begin with the formal T-matrix equation (for a regular potential), which is written as:

$$
\begin{aligned}
T(E) & =V+V G_{0}^{(+)}(E) T(E)=\left[1-V G_{0}^{(+)}(E)\right]^{-1} V \\
& =V+T(E) G_{0}^{(+)}(E) V=V\left[1-G_{0}^{(+)}(E) V\right]^{-1}
\end{aligned}
$$

where the free Green's function for the two-body system, with the appropriate boundary condition, in terms of the free Hamiltonian $H_{0}$, is

$$
G_{0}^{(+)}(E)=\left(E+i \varepsilon-H_{0}\right)^{-1}
$$

Considering that our input is given by the T-matrix at a given scale, the potential will formally be replaced by the T-matrix at scale $\mu$ through Eq.(3) or Eq.(4):

$$
V=T\left(-\mu^{2}\right)\left[1+G_{0}\left(-\mu^{2}\right) T\left(-\mu^{2}\right)\right]^{-1}=\left[1+T\left(-\mu^{2}\right) G_{0}\left(-\mu^{2}\right)\right]^{-1} T\left(-\mu^{2}\right) .
$$

Substituting the potential given by Eq.(6) in Eqs. (3) and (4), the corresponding subtracted equation for the T-matrix is found:

$$
T(E)=T\left(-\mu^{2}\right)+T\left(-\mu^{2}\right)\left(G_{0}^{(+)}(E)-G_{0}\left(-\mu^{2}\right)\right) T(E)
$$

One should note that we have chosen a real value for $\mu$ (negative energy), but this is not a restriction to the generality of the subtracted T-matrix equation (7). However, such choice has the advantage that the kernel of the integral equation has just one fixed point singularity for positive energies $E$, and the same formal structure as the original T-matrix equation. As one can see, from the original T-matrix, one simply needs to replace $V$ by $T\left(-\mu^{2}\right)$ and multiply the free propagator by a function which depends on the scale $\mu$, such that

$$
T(E)=T\left(-\mu^{2}\right)+T\left(-\mu^{2}\right) G_{R}^{(+)}\left(E ;-\mu^{2}\right) T(E)
$$

where

$$
G_{R}^{(+)}\left(E ;-\mu^{2}\right) \equiv G_{0}^{(+)}(E)-G_{0}\left(-\mu^{2}\right)=\frac{\left(\mu^{2}+E\right)}{\left(\mu^{2}+H_{0}\right)} G_{0}^{(+)}(E)
$$

The renormalized T-matrix equation, given above, has a kernel which is subtracted at the energy point $-\mu^{2}$. Although the interaction $V$, as given by Eq. (6), is not defined 
for a singular interaction, the subtracted T-matrix equation is well defined for a regular interaction plus a delta potential. This justifies a posteriori the formal manipulations in obtaing Eq.(7). One could instead, work with a finite cut-off in the momentum integrations and derive the subtracted equation for the T-matrix; then take the infinite limit of the cut-off. Of course the subtracted equation for the T-matrix is insensible to the cut-off as long the potential is singular like a delta interaction.

In the second term of the right-hand side of Eq.(7), the propagation over the intermediate states is subtracted at an energy scale of $-\mu^{2}$. The physical information apparently lost in this subtraction is contained in $T\left(-\mu^{2}\right)$, which in the case of pure contact interaction is the renormalized coupling constant [13].

If one sets $T\left(-\mu^{2}\right)$ equal to a given operator ( $\mu$ independent), the scheme becomes dependent on the subtraction point, which only has $\mu$ vanishing as a physical parameter (i.e., the theory is renormalization group invariant) in the limit $\mu \rightarrow \infty$. This is a trivial observation when such operator is a regular potential. Thus, setting the potential as equal to the T-matrix at $E=-\mu^{2}$, the present scheme implies, that the physical T-matrix is found in the limit $\mu \rightarrow \infty$. We will show that the procedure can be applied for a singular potential like OPEP plus contact term, and the limit $\mu \rightarrow \infty$ gives finite physical observables with vanishing dependence on $\mu$. The numerical results obtained by constraining $T\left(-\mu^{2}\right)$ to be equal to OPEP plus contact term for several values of $\mu$ show quantitatively the limiting process, while the scattering lengths are kept fixed. In this way, one can also verify how good is a scale as low as the $\rho$-meson mass, in order to approach the limiting values for the observables. Anticipating our numerical findings, the low-energy phase-shifts for the ${ }^{1} S_{0},{ }^{3} S_{1}$ and ${ }^{3} D_{1}$ channels give a reasonable support to such low value of $\mu$. However, this is not true for the $\epsilon_{1}$ mixing-parameter, even for energies as low as $20 \mathrm{MeV}$.

Let us apply the subtracted T-matrix equation for the pure contact interaction, and reobtain some well-known results just for the sake of completeness. In this case, the matrix elements of the T-matrix are independent of the momentum and, at $E=-\mu^{2}$, it is given by the renormalized coupling constant:

$$
\left\langle\overrightarrow{p^{\prime}}\left|T\left(-\mu^{2}\right)\right| \vec{p}\right\rangle=\frac{1}{2 \pi^{2}} \lambda_{\mathcal{R}}(\mu)
$$

The renormalized T-matrix in the subtraction point is given by $\lambda_{\mathcal{R}}(\mu)$, which is the physical information supplied by the two-body system in order to obtain other observables. For example, $\lambda_{\mathcal{R}}(0)$ is identified with the scattering length $(a), \lambda_{\mathcal{R}}(0)=a$. Solving Eq.(7), in the usual three-dimensional space with condition (10),

$$
\left\langle\overrightarrow{p^{\prime}}|T(E)| \vec{p}\right\rangle=\left[\frac{2 \pi^{2}}{\lambda_{\mathcal{R}}(\mu)}-\int d^{3} q\left(\frac{1}{E+i \varepsilon-q^{2}}+\frac{1}{\mu^{2}+q^{2}}\right)\right]^{-1}
$$

The on-shell-momentum is $k=\sqrt{E}$ and 


$$
\left\langle\overrightarrow{p^{\prime}}|T(E)| \vec{p}\right\rangle=\frac{1}{2 \pi^{2}}\left[\frac{1}{\lambda_{\mathcal{R}}(\mu)}+(\mu+i k)\right]^{-1} .
$$

In general the matrix elements of a two-body interaction will depend on the momentum, spin and isospin, such that it will be usefull to define our notation for the partial wave decomposition. The decomposition of the plane-wave is given by

$$
\left|\vec{p} ; s m_{s} ; I\right\rangle=\sqrt{\frac{2}{\pi}} \sum_{l s j m_{j} ; I}\left|p ; l s ; j m_{j}\right\rangle|I\rangle\left[\mathcal{Y}_{l s}^{j m_{j}}(\hat{p})\right]^{\dagger}\left|s m_{s}\right\rangle
$$

where the quantum numbers $j, m_{j}, l, s, I$ refer, respectively, to the two-body operators: total angular momentum $\mathbf{J}$, its z-component $J_{z}$, orbital angular momentum $\mathbf{L}$, total spin $\mathbf{S}$ and total isospin I. $\mathcal{Y}_{l_{s}}^{j m_{j}}(\hat{p})$ are the usual orthonormalized functions for the coupled angular $(\mathbf{L})$ and spin $(\mathbf{S})$ momentum to the total momentum $(\mathbf{J}=\mathbf{L}+\mathbf{S}) \cdot \hat{p}$ defines the two-dimensional angular variables related to the vector $\vec{p}$.

The partial wave decomposition of OPEP (2) and, for simplicity, dropping the unnecessary indices for the total angular momentum and spin in $V_{\pi}$, is given by

$$
\begin{aligned}
V_{\pi, \alpha}^{\left(l^{\prime} l\right)}\left(p^{\prime}, p\right)= & -\frac{g_{a}^{2}\left[2 I_{\alpha}\left(I_{\alpha}+1\right)-3\right]}{(8 \pi)^{2} f_{\pi}^{2}} \int d \hat{p} \int d \hat{p}^{\prime}\left(\mathcal{Y}_{l \frac{1}{2}}^{j m_{j}}(\hat{p})\right)^{\dagger} \times \\
& {\left[\frac{\overrightarrow{\sigma_{1}} \cdot\left(\overrightarrow{p^{\prime}}-\vec{p}\right) \vec{\sigma}_{2} \cdot\left(\overrightarrow{p^{\prime}}-\vec{p}\right)}{\left(\overrightarrow{p^{\prime}}-\vec{p}\right)^{2}+m_{\pi}^{2}}\right]\left(\mathcal{Y}_{l^{\prime} \frac{1}{2}}^{j m_{j}}\left(\hat{p}^{\prime}\right)\right), }
\end{aligned}
$$

where for the singlet state $\alpha=s\left(I_{s}=1\right)$ and for triplet state $\alpha=t\left(I_{t}=0\right)$.

So, in the angular projected equation for the T-matrix, the singlet ${ }^{1} S_{0}$ state matrix element for the OPEP, corresponding to eq. (2), is given as

$$
V_{\pi, s}^{(00)}\left(p^{\prime}, p\right)=\frac{g_{a}^{2}}{32 \pi f_{\pi}^{2}}\left(2-\int_{-1}^{1} d x \frac{m_{\pi}^{2}}{p^{2}+p^{\prime 2}-2 p p^{\prime} x+m_{\pi}^{2}}\right) .
$$

In the limit of $p$ or $p^{\prime}$ going to infinity the matrix element goes to a constant:

$$
\lim _{p^{\prime} \rightarrow \infty} V_{\pi, s}^{(00)}\left(p^{\prime}, p\right)=\frac{g_{a}^{2}}{16 \pi f_{\pi}^{2}} .
$$

The corresponding angular momentum projected matrix elements, for triplet ${ }^{3} S_{1}-{ }^{3} D_{1}$ coupled channels, $\left(V_{\pi, t}^{\left(l^{\prime} l\right)}\right.$, with $\left.l^{\prime}, l=0,2\right)$ are: 


$$
\begin{aligned}
& V_{\pi, t}^{(0,0)}\left(p^{\prime}, p\right)=\frac{g_{a}^{2}}{32 \pi f_{\pi}^{2}}\left(2-\int_{-1}^{1} d x \frac{m_{\pi}^{2}}{p^{2}+p^{\prime 2}-2 p p^{\prime} x+m_{\pi}^{2}}\right), \\
& V_{\pi, t}^{(20)}\left(p^{\prime}, p\right)=\frac{g_{a}^{2} \sqrt{2}}{16 \pi f_{\pi}^{2}} \int_{-1}^{1} d x \frac{p^{\prime 2}-2 p p^{\prime} x+p^{2}\left(\frac{3}{2} x^{2}-\frac{1}{2}\right)}{p^{2}+p^{\prime 2}-2 p p^{\prime} x+m_{\pi}^{2}} \\
& V_{\pi, t}^{(22)}\left(p^{\prime}, p\right)=\frac{g_{a}^{2}}{32 \pi f_{\pi}^{2}} \int_{-1}^{1} d x \frac{2 p p^{\prime} x-\left(p^{2}+p^{2}\right)\left(\frac{3}{2} x^{2}-\frac{1}{2}\right)}{p^{2}+p^{\prime 2}-2 p p^{\prime} x+m_{\pi}^{2}}
\end{aligned}
$$

and $V_{\pi, t}^{(02)}\left(p^{\prime}, p\right)=V_{\pi, t}^{(20)}\left(p, p^{\prime}\right)$.

In the limit of $p$ or $p^{\prime}$ going to infinity, the matrix elements of OPEP in the ${ }^{3} S_{1}-{ }^{3} D_{1}$ channels are zero or constant:

$$
\begin{array}{ll}
\lim _{p^{\prime} \rightarrow \infty} V_{\pi, t}^{(00)}\left(p^{\prime}, p\right)=\frac{g_{a}^{2}}{16 \pi f_{\pi}^{2}}, & \lim _{p^{\prime} \rightarrow \infty} V_{\pi, t}^{(22)}\left(p^{\prime}, p\right)=0, \\
\lim _{p^{\prime} \rightarrow \infty} V_{\pi, t}^{(20)}\left(p^{\prime}, p\right)=\frac{g_{a}^{2} \sqrt{2}}{8 \pi f_{\pi}^{2}}, & \lim _{p \rightarrow \infty} V_{\pi, t}^{(20)}\left(p^{\prime}, p\right)=0
\end{array}
$$

consequently, just one subtraction is enough to obtain a finite T-matrix, once $T\left(-\mu^{2}\right)$ is given.

The trivial choice for $T\left(-\mu^{2}\right)$ is the OPEP operator added to a delta interaction. For the singlet renormalized T-matrix, with the renormalized strength of the contact interaction $\left(\lambda_{\mathcal{R}, s}(\mu)\right)$ fixed by the singlet scattering length $a_{s}=-23.7 \mathrm{fm}$, we have

$$
T_{s}^{(00)}\left(p^{\prime}, p ;-\mu^{2}\right)=V_{\pi, s}^{(00)}\left(p^{\prime}, p\right)+\lambda_{\mathcal{R}, s}(\mu),
$$

where we have dropped the upper index in $\lambda$ for simplicity. As for the coupled ${ }^{3} S_{1}-{ }^{3} D_{1}$ channels, the renormalized T-matrix for each channel $\left(l, l^{\prime}=0,2\right)$ is given by

$$
T_{t}^{\left(l^{\prime} l\right)}\left(p^{\prime}, p ;-\mu^{2}\right)=V_{\pi, t}^{\left(l^{\prime} l\right)}\left(p^{\prime}, p\right)+\lambda_{\mathcal{R}, t}(\mu) \delta_{l^{\prime} 0} \delta_{l 0}
$$

In this case, the renormalized strength of the contact interaction $\left(\lambda_{\mathcal{R}, t}(\mu)\right)$ is fixed by the triplet scattering length, $a_{t}=5.4 \mathrm{fm}$.

Next, the formalism in momentum space, for the renormalized partial-wave T-matrix, using one subtraction in the propagator at an energy $-\mu^{2}$ is concluded by writting the integral equations corresponding to Eq. (8) where one should replace the matrix elements for $T\left(-\mu^{2}\right)$ by the corresponding above expressions [for the singlet state, eq. (21); for the triplet state, eq. (22)]. For the singlet state, we have

$$
T_{s}^{(00)}\left(p^{\prime}, p ; k^{2}\right)=T_{s}^{(00)}\left(p^{\prime}, p ;-\mu^{2}\right)+
$$




$$
\frac{2}{\pi} \int_{0}^{\infty} d q q^{2}\left(\frac{\mu^{2}+k^{2}}{\mu^{2}+q^{2}}\right) \frac{T_{s}^{(00)}\left(p^{\prime}, q ;-\mu^{2}\right)}{k^{2}-q^{2}+i \epsilon} T_{s}^{(00)}\left(p^{\prime}, p ; k^{2}\right)
$$

and for the triplet state, with $l_{i}=0,2(i=1,2,3)$, the corresponding coupled equation is given by:

$$
\begin{aligned}
T_{t}^{\left(l_{1} l_{2}\right)}\left(p^{\prime}, p ; k^{2}\right)= & T_{t}^{\left(l_{1} l_{2}\right)}\left(p^{\prime}, p ;-\mu^{2}\right)+ \\
& \frac{2}{\pi} \sum_{l_{3}} \int_{0}^{\infty} d q q^{2}\left(\frac{\mu^{2}+k^{2}}{\mu^{2}+q^{2}}\right) \frac{T_{t}^{\left(l_{1} l_{3}\right)}\left(p^{\prime}, q ;-\mu^{2}\right)}{k^{2}-q^{2}+i \epsilon} T_{t}^{\left(l_{3} l_{2}\right)}\left(p^{\prime}, p ; k^{2}\right)
\end{aligned}
$$

These eqs. (23) and (24) for a regular potential clearly converge to the exact original T-matrix in the limit $\mu^{2} \rightarrow \infty$, with the corresponding Born terms given by the potential.

\section{Numerical results and conclusions}

In the following, we present the numerical results for the observables, in the ${ }^{1} S_{0}$ and ${ }^{3} S_{1}-{ }^{3} D_{1}$ channels, calculated from the leading order NN interaction, with the subtracted eq. (7) and the T-matrix elements at scale $\mu$ defined by eqs. (21) and (22). Distinct numerical procedures can be used to obtain the final results, from the equations (23) and (24). In our numerical calculation, we have followed the one described in Ref. ([14]), where the T-matrix is related to an auxiliary real matrix, which is solved by the inverse matrix approach. Considered that in the present work we are not interested in high numerical accuracy, any of the usual numerical procedures should give about the same results we obtain, once a large enough number of points is taken in the numerical discretization of the momentum space. In our calculation, when discretizing the momentum space $(p)$ from 0 to $\infty$, we have used a Gaussian mesh with up to 100 points, mapping the interval $[-1,1]$ to $[0, \infty]$ through the expression $p=p_{0}(1+x) /(1-x) \cdot p_{0}$ is adjusted to optimize the convergence of the results, and in practice should be comparable with $\mu$, when a minimum number of mesh points is required.

In Table I, for several values of $\mu$, we show the corresponding results for the singlet and triplet effective ranges, and also for deuteron observables (binding energies and ratio $\eta_{D}$ ). The ratio $\eta_{D}$ is given by

$$
\eta_{D}=\lim _{k \rightarrow i k_{B}} \frac{T_{t}^{(02)}\left(k, k ; k^{2}\right)}{T_{t}^{(00)}\left(k, k ; k^{2}\right)}
$$

where the deuteron binding energy is $E=-k_{B}^{2}$. The effective ranges, for the singlet state $\left(r_{0, s}\right)$ and for the triplet state $\left(r_{0, t}\right)$ and the corresponding scattering lengths $\left(a_{s}\right.$ and $\left.a_{t}\right)$ are related to the $s$-wave phase shifts, according to the effective range expansion: 


$$
k \cot \left(\delta_{0, \alpha}^{(\alpha)}(k)\right)=-\frac{1}{a_{\alpha}}+\frac{1}{2} r_{0, \alpha} k^{2}+\mathcal{O}\left(k^{4}\right) .
$$

Table I Low energy n-p and deuteron observables compared with data. Singlet $\left(r_{0, s}\right)$ and triplet $\left(r_{0, t}\right)$ effective ranges, deuteron binding energy $\left(B_{D}\right)$ and ratio $\eta_{D}$ are given for several values of the single parameter $\mu$ which is used in our renormalization scheme. The $\lambda_{\mathcal{R}, s}(\mu)$ and $\lambda_{\mathcal{R}, t}(\mu)$ are the strenghts of the $\delta$-interactions which were added to the OPEP and adjusted to the corresponding scattering lengths, $a_{s}=-23.7 \mathrm{fm}$ and $a_{t}=5.4 \mathrm{fm}$. The results were compared with data obtained from Ref. [15].

\begin{tabular}{ccccccc}
\hline \hline$\mu\left(\mathrm{fm}^{-1}\right)$ & $\lambda_{\mathcal{R}, s}(\mathrm{fm})$ & $r_{0, s}(\mathrm{fm})$ & $\lambda_{\mathcal{R}, t}(\mathrm{fm})$ & $r_{0, t}(\mathrm{fm})$ & $B_{D}(\mathrm{MeV})$ & $\eta_{D}$ \\
\hline 4 & -0.8806 & 1.332 & -0.2281 & 1.364 & 1.977 & 0.02808 \\
10 & -0.7570 & 1.345 & 21.741 & 1.536 & 2.084 & 0.02904 \\
30 & -0.6977 & 1.347 & -0.3776 & 1.582 & 2.114 & 0.02933 \\
Ref.[15] & - & 2.73 & - & 1.75 & 2.2246 & 0.0256 \\
\hline \hline
\end{tabular}

The results in Table I are compared with experimental results obtained from Ref.[15], and show a good qualitative agreement, considering that we are only using the leading order term of the effective $\mathrm{NN}$ interaction. As it is shown, the agreement improves as we increase the value of $\mu$. The results in the ${ }^{1} S_{0}$ channel could be improved by adding higher-order terms in the effective interaction, as already one can deduce from previous approaches used by other authors in EFT $[4,7,8]$. It is also important to observe that for $\mu>4 \mathrm{fm}^{-1}, r_{0, s}$ is pratically stable, such that it is insensible to nucleon propagation for higher momenta once the scattering length is fixed. The insensibility to $\mu>4 \mathrm{fm}^{-1}$ is also found in the singlet phase-shifts up to laboratory energies of about $100 \mathrm{MeV}$.

In the triplet channel, we observe a large variation of $\lambda_{\mathcal{R}, t}(\mu)$ as the scale is moved to higher values. The oscillating behaviour is a consequence of the zeroes of $\lambda_{\mathcal{R}, t}(\mu)$ at those scales where only OPEP is enough to reproduce the triplet scattering length. However, in spite of such variation, the triplet low-energy and deuteron observables are quite stable, and converging in the limit of $\mu$ going to infinity. From the observables we have examined, as we pointed out in our discussion, the slowest rate of convergence is found for the mixing parameter $\epsilon_{1}$, which is defined through the on-shell $\mathrm{S}-$ and $\mathrm{T}$-matrices in the coupled channel. With an implicit energy dependence, such relation can be written as a two-dimensional matrix and parametrized according to Ref. [16]: 


$$
S=1-2 i k T_{t}=\left(\begin{array}{cc}
\cos \left(2 \epsilon_{1}\right) e^{2 i \delta_{0, t}} & i \sin \left(2 \epsilon_{1}\right) e^{i\left(\delta_{0, t}+\delta_{2, t}\right)} \\
i \sin \left(2 \epsilon_{1}\right) e^{i\left(\delta_{0, t}+\delta_{2, t}\right)} & \cos \left(2 \epsilon_{1}\right) e^{2 i \delta_{2, t}}
\end{array}\right)
$$

In Figs. 1, 2 and 3 we show the results for the neutron-proton phase-shifts ${ }^{1} S_{0},{ }^{3} S_{1}$ and ${ }^{3} D_{1}$, respectively. The results shown in Fig. 1 are pratically insensible to nucleon-nucleon propagation for momenta higher than $4 \mathrm{fm}^{-1}$, in agreement with results shown for the singlet effective range (Table I), such that no improvement can be expected for the singlet observables with just one subtraction/parameter. In Fig. 2, the good qualitative fit to data obtained with the scaling parameter $\mu=4 \mathrm{fm}^{-1}$ is improved when taking larger value for $\mu$. The subtraction point above $\mu>4 \mathrm{fm}^{-1}$ has some effect, as also anticipated from the values of scattering length and effective range (see Table I); but the effect is not strong above $30 \mathrm{fm}^{-1}$. In Fig. 3, we observe that the insensibility to nucleon-nucleon propagation at higher momenta is also found in the $D$-wave. In this state the phase-shift is clearly dominated by the OPEP, as also shown in ref. [10], in a first order perturbative calculation.

In Fig. 4 we present our results for the mixing parameter, $\epsilon_{1}$, which is obtained using eq. (27). The results are insentive to $\mu>4 \mathrm{fm}^{-1}$ only for $E_{l a b}<10 \mathrm{MeV}$, and show a fair reproduction of the Nijmegen values. This follows due to the dominance of OPEP in the slope of $\epsilon_{1}$ at zero energy[17]. Above $10 \mathrm{MeV}$, the mixing parameter shows a dependence on the values of $\mu$, which is reduced by increasing $\mu$ above $30 \mathrm{fm}^{-1}$, while the agreement with data is improved as $\mu \rightarrow \infty$. The mixing parameter is the most sensitive parameter in respect to changes in the subtraction point for $E_{l a b}>10 \mathrm{MeV}$ and for $\mu$ between 4 and $30 \mathrm{fm}^{-1}$. Our results in the limit of $\mu \rightarrow \infty$ are consistent with the ones obtained by Ballot and Robillota [10], where they have used a cut-off parameter.

The results presented in Table I and Figs. 1-4, when compared with fitted data [15] and with other calculations with more fitting parameters [3,4,11], are a clear evidence of the dominance of the leading order term (OPEP) in low-energy NN observables. This implies that one should first renormalize the T-matrix for the leading order term and then add higher order terms to the NN interaction in the chiral expansion, or in the EFT expansion. Our results for the phase-shifts and mixing parameter show that the mixing parameter is the most sensitive observable to the subtraction point used in the renormalization procedure. The phase-shifts for ${ }^{1} S_{0}$ state and for the ${ }^{3} D_{1}$ are pratically insensitive to $\mu$ larger than $4 \mathrm{fm}^{-1}$, with an evidence that ${ }^{3} D_{1}$ is dominated by the OPEP, in agreement with previous calculations[10]. The present results confirm that the addition of higher order singular terms in the potential are needed in order to fit the singlet effective range and corresponding phase-shifts. This observation was already verified in several EFT approaches $[3-7,11,12]$, without however addressing to the full predictibility of the leading order term. This lead some authors to affect negatively the nice fitting obtained for the triplet channel when trying to improve the observables for the singlet channel [4]. In order to avoid this problem, it is important to parametrize the higher order corrections, such that it affects mostly the singlet channel (where the leading order fails to give a good 
description also to the effective range, independently of the renormalization point). In the ${ }^{3} S_{1}-{ }^{3} D_{1}$ channels, the higher order terms will become more important for laboratory energies above $50 \mathrm{MeV}$, where the leading order results deviate from the data (as shown in Figs. 2 and 4).

Considering the results obtained and shown in Figs. 1-4, we would like to make some comments about the renormalization scale $\mu$, which appears in our approach as the point where we introduce a subtraction in the nucleon-nucleon propagator. (This parameter is directly related to the "cut-off" parameter of other approaches.) Weinberg [1] has originally suggested to cut the virtual propagation of the NN system at the scale $m_{\rho}$, which implies $\mu \sim 4 \mathrm{fm}^{-1}$, and this suggestion is consistent with our results, as it is almost exactly verified for the ${ }^{1} S_{0}$ and ${ }^{3} D_{1}$ phase shifts (see Figs. 1 and 3), and also approximately for the ${ }^{3} S_{1}$ phase shifts (Fig. 2). We further observe that our results for $\mu \rightarrow \infty$ (numerically, we have used $\mu \leq 60 \mathrm{fm}^{-1}$ ) are about the same we got for the ${ }^{1} S_{0}$ and ${ }^{3} D_{1}$, and improves the results we obtain for the ${ }^{3} S_{1}$ case, for the mixing parameter, and also related observables, as shown in Table I. Such results, particularly the results for the mixing parameter, lead us to conclude that Weinberg's suggestion can only be understood as a qualitative statement, and as such, it is verified. However, we observe that the mixing parameter, which is very sensible to this renormalization scale, is violating this statement even for $E_{l a b}>20 \mathrm{MeV}$. This result implies that some essential physical information for such observable is being lost when using $\mu \sim m_{\rho}$, and as we observe for other observables, there is no danger in increasing the value of $\mu$. Another important observation, related to a statement of caution made in ref.[7], is that we are not unnecessarily complicating the theory, or introducing irrelevant degrees of freedom when considering $\mu \rightarrow \infty$, as it is possible to occur in other EFT approaches. Our conclusion for a practical calculation with the leading order term is that one should use a momentum renormalization parameter of the order of $\sim 4 \mathrm{fm}^{-1}$ (as suggested in [1]) to obtain qualitative results for the low energy NN observables. But, to improve the results for some observables, like the triplet mixing parameter shown in Fig. 4 and also the ${ }^{3} S_{1}$ phase shifts, there is no danger in extending $\mu \rightarrow \infty$, as the previous results are not affected by increasing such value for the scale.

In conclusion, we have examined the complete renormalization of the leading order term of the neutron-proton interaction. We suggest the use of a T-matrix with a subtracted propagator, at an energy $-\mu^{2}$, and we have concluded about an overall insensitivity of the low-energy singlet and triplet phase-shifts for momentum scale $\mu$ larger than $4 \mathrm{fm}^{-1}$. Such result supports the qualitative suggestion presented in Ref. [1]. However, the results for the mixing parameter (see Fig. 4) show that this observable is more sensible to the renormalization scale, and therefore more appropriate than the diagonal phase-shift results to be described by an EFT. Our results for this observable show that $\mu \rightarrow \infty$ is necessary to have a better agreement with data. And notice that a larger value of $\mu$ is also consistent with the previous results obtained for the phase-shifts. We can conclude that, in our renormalization scheme, $\mu$ vanishes as a physical parameter, and the theory is renormalization group invariant in the limit $\mu \rightarrow \infty[2$.

\footnotetext{
2 By excluding the non-diagonal matrix element one could reach a wrong conclusion about $\mu$.
} 
It has been achieved with this work that the renormalized T-matrix for the leading order interaction has a well defined limit for the physical observables for $\mu \rightarrow \infty$ with the constraint that $T\left(-\mu^{2}\right)$ being equal to OPEP plus contact interaction, which also is supported by the data in the triplet channel. The following step to be investigated is the inclusion of the next order term in the EFT expansion of the NN interaction, i.e., the Laplacian of the delta potential. In this case, more subtractions are required to construct the renormalized T-matrix, starting from the T-matrix (for the leading order) obtained in this work.

We thank Drs. A. Delfino, M. Malheiro and P. Sauer for useful discussions. This work was partially supported by Fundação de Amparo à Pesquisa do Estado de São Paulo (FAPESP) and Conselho Nacional de Desenvolvimento Científico e Tecnológico (CNPq).

\section{References}

[1] S. Weinberg, Nucl. Phys. B 363 (1991) 2; Phys. Lett. B 295 (1992) 114.

[2] G.P. Lepage, How to renormalize the Schrödinger equation, proceedings of the VIII Jorge André Swieca Summer School Nuclear Physics, edited by C.A. Bertulani, M.E. Bracco, B.V. Carlson, and M. Nielsen (World Scientific, Singapore, 1997), pg. 135.

[3] C. Ordóñez, L. Ray, U. van Kolck, Phys. Rev. Lett. 72 (1994) 1982; C. Ordóñez, L. Ray, U. van Kolck, Phys. Rev. C 53 (1996) 2086.

[4] D.B. Kaplan, M.J. Sawage and M.D. Wise, Phys. Lett. B 424 (1998) 396; D.B. Kaplan, Nucl. Phys. B 478 (1996) 629.

[5] E. Epelbaoum, W. Glöckle, A. Kruger, and Ulf-G. Meissner, Nucl. Phys. A 645 (1999) 413; E. Epelbaoum, W. Glöckle, and Ulf-G. Meissner, Phys. Lett. B 439 (1998) 1; Ulf-G. Meissner, Acta Phys. Polon. B 29 (1998) 2339.

[6] K.G. Richardson, M.C. Birse, and J.A. McGovern, Renormalization and power counting in effective field theories for nucleon-nucleon scattering, hep-ph/9708435; M.C. Birse, Renormalising NN scattering: is power counting powerless?, nucl-th/9804028; M.C. Birse, J.A. McGovern, K.G. Richardson, The Wilson Renormalization group applied to the potential in NN scattering, hep-ph/9808398; D.R. Phillips, S.R. Beane, M.C. Birse, Scheming in Dimensional regularization, hep-th/9810049;

[7] T.-S. Park, K. Kubodera, D.-P. Min, M. Rho, Phys. Rev. C 58 (1998) 637.

[8] T. Mehen and I.W. Stewart, Phys. Lett. B 445 (1999) 378.

[9] R.J. Perry and S. Szpigel, A new renormalization group for Hamiltonian field theory, nuclth/9901079.

[10] J.L. Ballot and M.R. Robilotta, J.Phys. G 20 (1994) 1599.

[11] T.D. Cohen, Phys. Rev. C 56 (1997) 679; D.R. Phillips and T.D. Cohen, Phys. Lett. B 390 (1997) 7. 
[12] J. Gegelia, Phys. Lett. B 429 (1998) 227; Are pions perturbative in effective field theory?, nucl-th/9806028; About the equivalence of cut-off and renormalised effective field theories, nucl-th/9805008.

[13] S.K. Adhikari, T.Frederico, and I.D. Goldman, Phys. Rev. Lett. 74 (1995) 487; S.K. Adhikari and T. Frederico Phys. Rev. Lett. 74 (1995) 4572; C. F. de Araujo, L. Tomio, S. K. Adhikari, T. Frederico, J. Phys. A30, 4687 (1997). A recent pedagogical introduction to renormalization in elementary quantum mechanics is given in I. Mitra, A. DasGupta, and B. Dutta-Roy, Am. J. Phys. 66 (1998) 1101, and references therein.

[14] L. Tomio and S.K. Adhikari, Phys. Rev. C 22 (1980) 28.

[15] V. G. J. Stocks, R.A.M. Klomp, C.P.F. Terheggen; and J.J. de Swart, Phys. Rev. C 49 (1994) 2950; V.G.J. Stocks, R.A.M. Klomp, M.C.M. Rentmeester and J.J. de Swart, Phys. Rev. C 48 (1993) 792.

[16] H.P. Stapp, T.J. Ypsilantis, and N. Metropolis, Phys. Rev. 105 (1957) 302.

[17] S.K. Adhikari, L.Tomio, J.P.B.C. de Melo and T. Frederico, Phys. Lett. B 318 (1993) 14; S.K. Adhikari, C.F. de Araujo and T. Frederico, Phys. Rev. C 50 (1994) R2684. 


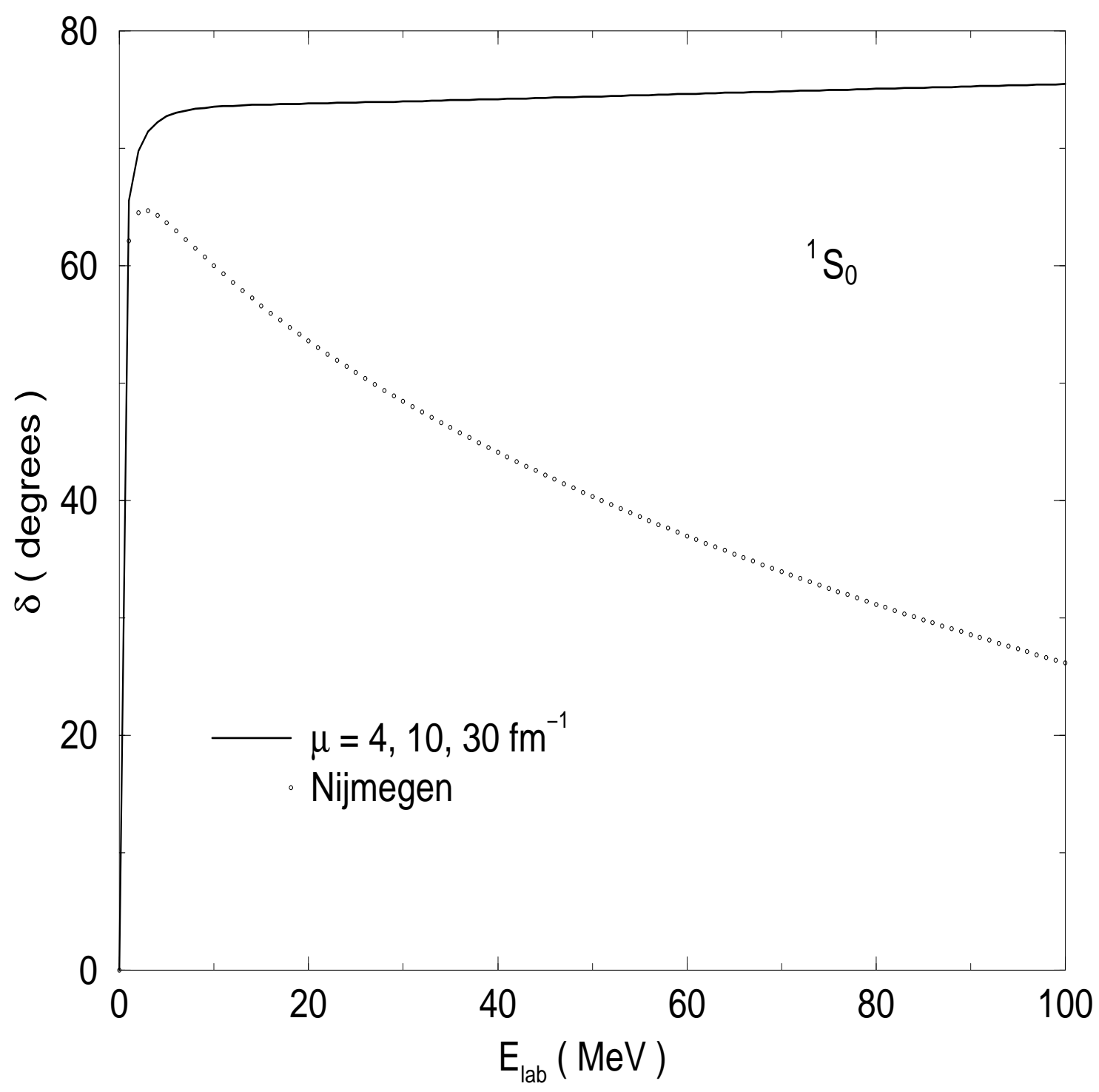

Fig. 1. Neutron-Proton phase shifts for the ${ }^{1} S_{0}$ state, $\delta_{0, s}$. The solid line is the model result, for different parameters $\mu$; the dotted line gives the fitting to data from Ref. [15]. 


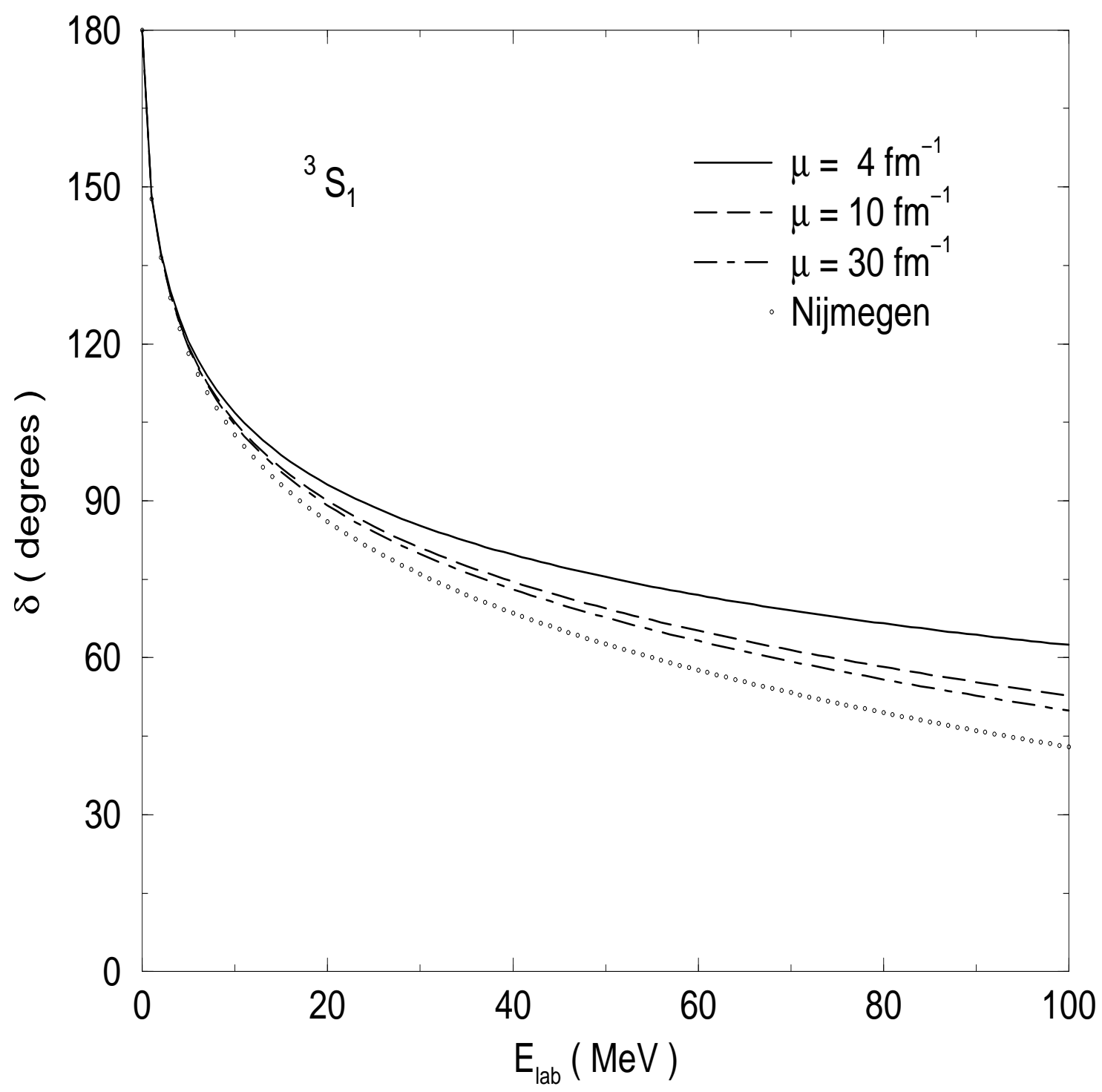

Fig. 2. Neutron-Proton phase shifts for the ${ }^{3} S_{1}$ state, $\delta_{0, t}$. Calculations are presented for $\mu=4$, 10 and $30 \mathrm{fm}^{-1}$; the dotted line gives the fitting to data from Ref. [15]. 


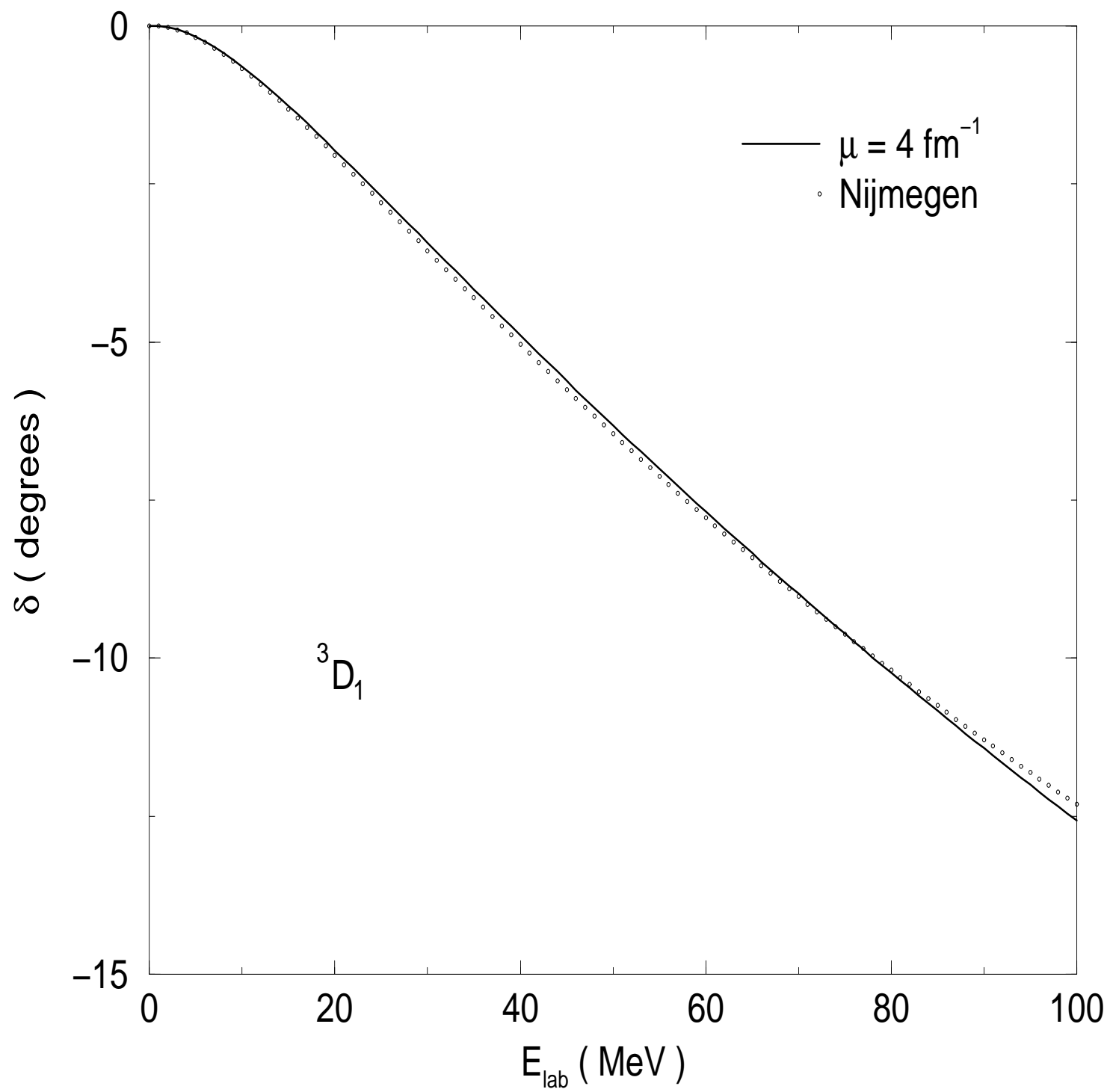

Fig. 3. Neutron-Proton phase shifts for ${ }^{3} D_{1}$ state, $\delta_{2, t}$. The solid line is the present calculation, for different parameters $\mu \geq 4 \mathrm{fm}^{-1}$; the dotted line gives the fitting to data from Ref. [15]. 


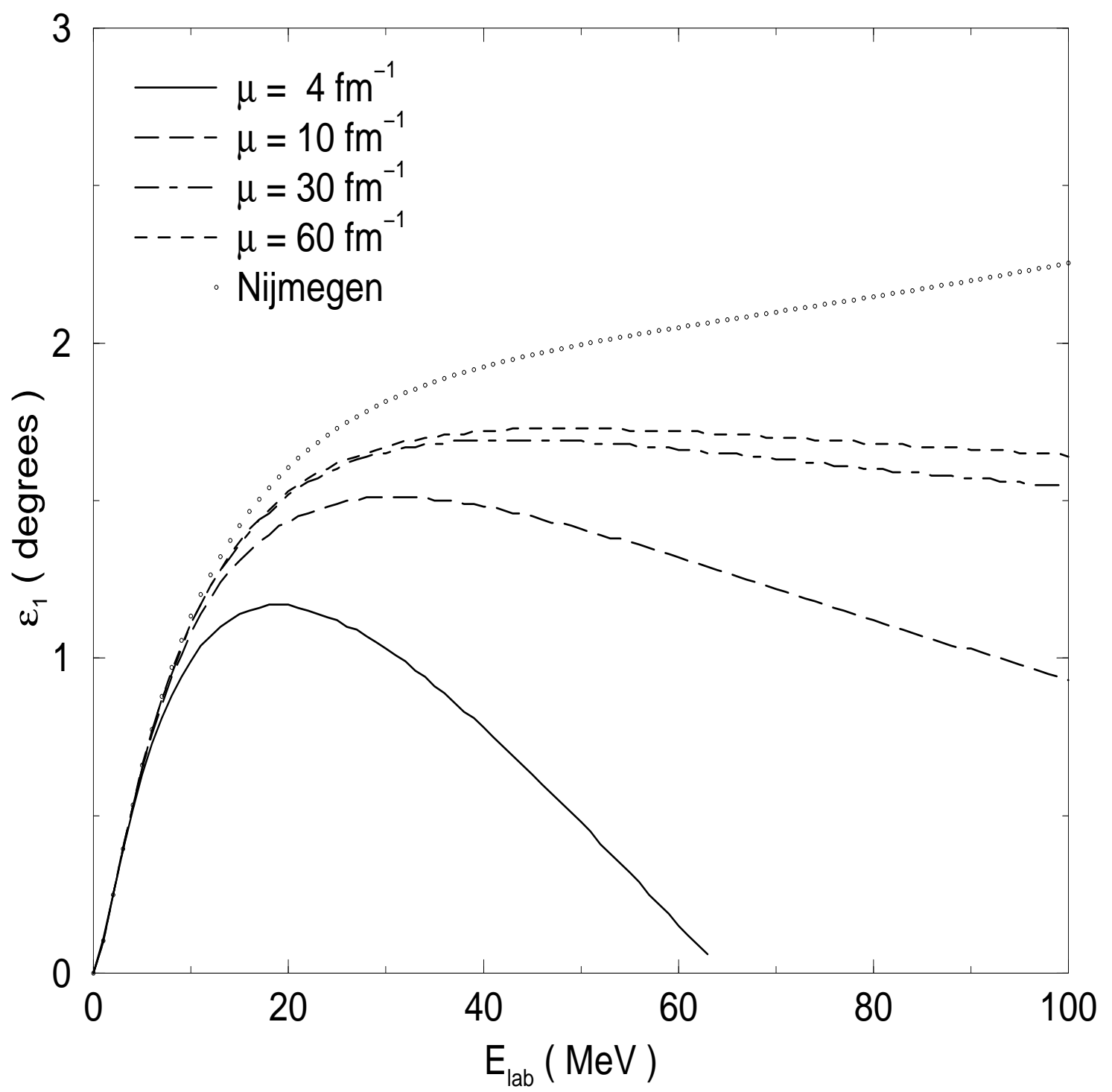

Fig. 4. Results for the mixing parameter (in degrees) given by eq. (27), for $\mu=4,10,30$ and 60 $\mathrm{fm}^{-1}$. The dotted line gives the fitting to data from Ref. [15]. 